Original Research Article

\title{
Drug utilization study in OPD of a tertiary care hospital in a rural area of Jalna, Maharashtra, India by using WHO prescribing indicators
}

\author{
Shruti Chandra*, Imran Nawaz Khan, Mohd Mateenudin, Ajay Chandrakapure, Syed Maaz, \\ Faheem Mubin
}

\begin{abstract}
Department of Pharmacology, Maharashtra, India

Revised: 16 December 2017

*Correspondence to:

Dr. Shruti Chandra,

Email: dr.shruti1204@

gmail.com
\end{abstract} JIIU'S IIMSR, Warudi, Jalna,

Received: 11 December 2017

Accepted: 19 December 2017

Copyright: () the author(s), publisher and licensee Medip Academy. This is an openaccess article distributed under the terms of the Creative Commons Attribution NonCommercial License, which permits unrestricted noncommercial use, distribution, and reproduction in any medium, provided the original work is properly cited.

\begin{abstract}
Background: Drug utilization studies (DUS) are used as potential tool in the evaluation of healthcare system. DUS are conducted to facilitate the rational use of drugs in populations. The indicators of prescription in practice measure the performance of health care provider in several key dimensions related to appropriate use of drug. Therefore, the present study was undertaken to analyze the prescription written by doctors in a Tertiary Care Hospital in rural area.

Methods: Present study is a cross sectional, prospective and observational study. The study was conducted in a rural tertiary, teaching hospital at JIIU's Indian Institute of Medical Science and Research, Warudi, Taluka Badnapur from January 2017 to September 2017 for duration of 9 months. Data was obtained from 300 prescriptions. Data was analysed as per WHO prescribing indicators.

Results: Average number of drugs in the present study was found to be 2.85 . Percentage of drugs prescribed by generic name is $13.48 \%$. Percentage of encounters with an antibiotic prescribed $60 \%$. Percentage of encounters with an injection prescribed $4.33 \%$. Percentage of drugs prescribed from essential drugs list $72.91 \%$.

Conclusions: These types of studies help to design policy for rational use of drugs and motivation of physician for rational use of drugs.
\end{abstract}

Keywords: Drug utilization studies, Essential drugs, WHO prescribing indicators, Polypharmacy

\section{INTRODUCTION}

Now a day's drug utilization studies (DUS) are used as potential tool in the evaluation of healthcare system. Drug utilization studies are powerful tools to ascertain the role of drug in society. They create a sound socio-medical and health economic basis for healthcare decision making. ${ }^{1,2}$ The World Health Organization (WHO) defines drug utilization research as "the marketing, distribution, prescription and use of drugs in a society, with special emphasis on the resulting medical, social, and economic consequences." 3 The principal aim of drug utilization research is to facilitate the rational use of drugs in populations. For the individual patient, the rational use of a drug implies the prescription of a well-documented drug at an optimal dose, together with the correct information, at an affordable price. ${ }^{3}$ Conducting periodic studies of pattern of drug use in various hospital settings or patient populations is therefore essential to critically analyse the current hospital drug policies and to make recommendations based on various guidelines to improve upon the current drug usage pattern in the future, if needed. This is more importantly required in resource poor countries like ours so as to ensure that the scarce resources are utilized in the best possible manner. ${ }^{4,5}$ The indicators of prescription in practice measure the performances of health care provider in several key dimensions related to appropriate use of drug. Therefore, the present study was undertaken to analyze the prescriptions written by patients 
attending OPD of a teaching hospital in rural area. The indicators are based on the practices observed in a sample of clinical encounters taking place at outpatient health facilities for the treatment of acute or chronic diseases. The study was carried out in accordance with the WHO Prescribing indicators:

- $\quad$ Average number of drugs per encounter, measure the degree of polypharmacy.

- $\quad$ Percentage of drugs prescribed by generic name, to measure the tendency to prescribe by generic name.

- Percentage of encounters with an antibiotic prescribed.

- Percentage of encounters with an injection prescribed, to measure the overall level of use of two important, but commonly overused and costly forms of drug therapy.

- $\quad$ Percentage of drugs prescribed from essential drugs list, to measure the degree to which practices conform to a national drug policy, as indicated by prescribing from the national essential drugs list. ${ }^{6}$

\section{METHODS}

Present study was a cross sectional, prospective and observational study. The study was conducted in a rural area at JIIU's Indian Institute of Medical Science and Research, Warudi, Taluka Badnapur, Dist. Jalna from January 2017 to September 2017 for duration of 9 months. The study included prescriptions collected randomly from all OPDs. Prescriptions were scanned for the record purpose. Data was obtained from 300 prescriptions. Data was analyzed as per WHO prescribing indicators. Average number of drugs per encounter was calculated by dividing the total number of different drugs prescribed, by the number of encounters surveyed. It is not relevant whether the patient actually received the drugs. Percentage of drugs prescribed by generic name was calculated by dividing the number of drugs prescribed by generic name by the total number of drugs prescribed, multiplied by 100 .

Percentage of encounters with an antibiotic prescribed and Percentage of encounters with an injection prescribed were calculated by dividing the number of patient encounters during which an antibiotic or an injection are prescribed, by the total number of encounters surveyed, multiplied by 100. Percentage of drugs prescribed from essential drugs list was calculated by dividing the number of products prescribed which are listed on the essential drugs list by the total number of products prescribed, multiplied by 100 (WHO). ${ }^{6}$ For analysis of average number of drugs per encounter, combinations were considered as single drug and for the rest of indicators combination were split into individual drugs and counted separately.

Abbreviation or short forms used by doctors were not considered as generic for example: (Paracetamol written as PCM). Multivitamin prescription was counted as one, for example B-complex.

\section{RESULTS}

This study was performed as described earlier in the Material and methods with respect to the WHO recommended prescribing indicators. Indicators were calculated according to formula of described by WHO. ${ }^{6}$

Total number of drugs prescribed in 300 prescriptions was 853. Average number of drugs per encounter was 2.85 which is a reflection of Polypharmacy. This indicator shows that degree of Polypharmacy is low in our institute. Average number of drugs prescribed by Generic name was $13.48 \%$. Out of 853 drugs 115 drugs were prescribed by generic name which can be increased by motivation of practitioners. Only 13 prescriptions were found to have injections prescribed. Percentage of encounters with an injection prescribed was $4.33 \%$ which indicate judicious use of injections. 180 out of 300 prescriptions were found to have an antibiotic prescribed. Out of total 853 drugs, 622 drugs were found from essential list of medicines i.e. $72.91 \%$ which increases rationality of the prescription. In our institute good numbers of practitioners are using drugs from essential list of medicines.

Tabular representation of the prescribing indicators is given in Table 1. Figure 1 depicts prescribing trend.

Table 1: Prescribing indictors.

\begin{tabular}{|ll|}
\hline Indicator & Result \\
\hline Average number of drugs per encounter & 2.85 \\
\hline $\begin{array}{l}\text { Percentage of drugs prescribed by } \\
\text { generic name }\end{array}$ & $13.48 \%$ \\
\hline $\begin{array}{l}\text { Percentage of encounters with an } \\
\text { antibiotic prescribed }\end{array}$ & $60 \%$ \\
\hline $\begin{array}{l}\text { Percentage of encounters with an } \\
\text { injection prescribed }\end{array}$ & $4.33 \%$ \\
\hline $\begin{array}{l}\text { Percentage of drugs prescribed from } \\
\text { essential drugs list }\end{array}$ & $72.91 \%$ \\
\hline
\end{tabular}

Percentage of drugs prescribed by generic name

Percentage of encounters with an antibiotic prescribed

- Percentage of encounters with an injection prescribed

口Percentage of drugs prescribed from essential drug list

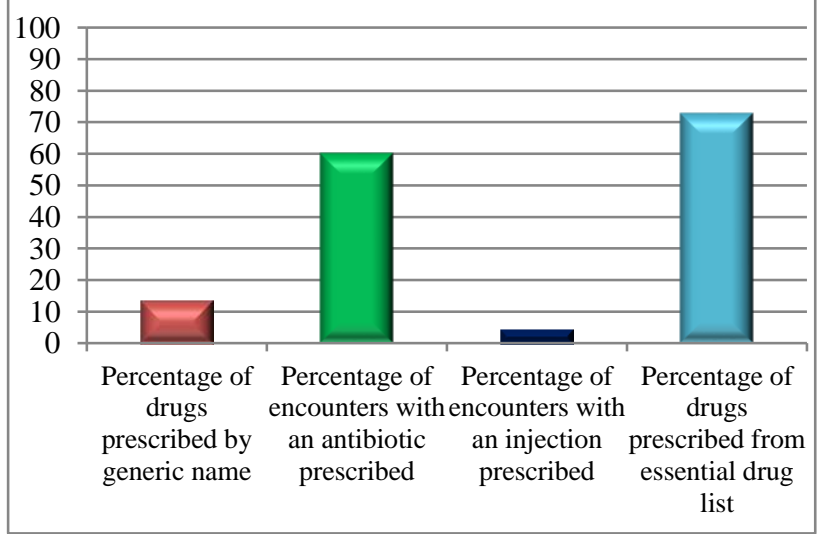

Figure 1: Prescribing trend. 


\section{DISCUSSION}

Average number of drugs per encounter in the present study was found to be 2.85 . In several other studies average number of drugs per encounter was (3.42), (2.3) and (2.22), (3.28) Simpson in non teaching clinicians and (2.82) in teaching clinicians Simpson. ${ }^{7-10}$ The findings of the present study are similar to that of teaching clinicians in the study by Simpson. ${ }^{10}$ The results of the present study is more as compared to (2.3) and (2.22), the results were less as compared to (3.42), non teaching clinicians 3.28 Simpson. ${ }^{7-10}$ Polypharmacy increases the cost of therapy unless the prescribed drugs are genuinely necessary. Also it decreases compliance of the patient so the drugs should be used judiciously. The risk of drug interaction increases from approximately $6 \%$ in patient taking two medication to $50 \%$ in those taking five medication to $100 \%$ in those taking 10 medication. ${ }^{11}$

Percentage of drug prescribed by generic name, in present study was $13.48 \%$. The results of several other studies were $(30.70 \%)$ while in a study conducted by Simpson it was $(5.75 \%)$ in teaching clinicians was $5.41 \% .^{7,10,12}$ The finding of the present study was less than Mohanty BK and was more than Ajapuje., ${ }^{72}$ Prescribing medicines by generic name avoid the confusion and makes therapy rational and cheaper. Moreover in the teaching institutions world over, in textbooks, in scientific journals and in the research publications, medicines are always mentioned by generic names. Despite this, most doctors prescribe the medicines by their brand names. The reason for this could be (1) tradition (2) aggressive medicine promotion (3) availability of multi-ingredient fixed dose drug combination. ${ }^{7}$ Encouraging prescriptions by generic names is always recommended by various national and international bodies to promote rational use of drugs. ${ }^{13}$ The drugs if prescribed by generic name also gives the advantage to the pharmacist to dispense the cheaper drugs to the patients, this helps to reduce the economic burden on patients. Also this practice will certainly help to check the luring practices if offered by some of the pharmaceutical companies to the practitioners for promoting their costlier brands. But implementation of this practice of prescribing by generic names is not always satisfactory and requires motivation of prescribers and strong regulatory interventions. ${ }^{13}$

The result of the present study showed that the percentage of encounters with an antibiotic prescribed was found to be $60 \%$. In several other studies, it was seen that percentage of encounters with antibiotic was $78.15 \%{ }^{7}$ Antibiotics should be judiciously used and when used, correct regimen should be followed. Injudicious use of antibiotic leads to development of antibiotic resistance. Antimicrobial resistance is a global problem and particularly pressing in developing countries where the infectious disease burden is high, and cost constrains the replacement of older antibiotics with newer more expensive ones. Based on studies of resistant infections, findings show that resistance level has been high. ${ }^{14}$
Irrational use of antibiotics can lead to emergence of antimicrobial resistant diseases, for example the recent emergence of Multi Drug Resistance Tuberculosis. WHO also has been trying to control the emergence of resistance to antibiotics and also spread the message for rational use of antibiotics. For this WHO on world health day i.e. April $7^{\text {th }}, 2011$ said "No action today, no cure tomorrow." 15 In our study $4.33 \%$ of encounters had an injection prescribed which is lower than $(10 \%)$ and another study conducted (14.10\%). ${ }^{16,17}$ Parenteral route (injection) should be used only when it is a must, otherwise it may become a vehicle for transmission of blood borne disease like Hepatitis B and C, HIV, Malaria etc. Also skilled person is required for such a route of administration of drug. Incidence of acute adverse drug reactions is more compared to oral route. It also increases the cost of therapy. ${ }^{14}$

WHO has defined "Essential medicines are those that satisfy the priority health care needs of the majority of population. ${ }^{10}$ In the present study, results showed that the percentage of drugs prescribed from essential drug list was (72.91\%), analysis was done with reference to Tripathi KD. ${ }^{18}$ In other study conducted by B.K. Mohanty results were $57.70 \%$ which is lower than ours it was reported that $77.61 \%$ drugs were from essential drug list which is higher than our results. ${ }^{12,19}$ Essential drugs should be preferred during prescribing. Prescription from the Essential drug list gives maximum benefit from limited resource, promotes rational use of drugs, assists the development of standard use of standard treatment protocol and rational prescribing policies and also increases economic advantages like lowering the cost of therapy. ${ }^{14}$

\section{CONCLUSION}

Thus, there is an ample scope of improving the prescribing pattern by keeping the number of medicines as low as possible, prescribing medicines by generic names, using medicines appropriately after selecting and consciously keeping the cost of therapy low. ${ }^{7}$ From above finding and results it can be concluded that such type of study will help in promoting, to design policy and motivation of physician for rational use of drugs.

\section{ACKNOWLEDGEMENTS}

Authors are thankful to Dr. A.B. Solepure, Dean, IIMSR, Warudi, Jalna for his valuable guidance and cooperation during the study.

\section{Funding: No funding sources \\ Conflict of interest: None declared \\ Ethical approval: The study was approved by the Institutional Ethics Committee}

\section{REFERENCES}

1. Bakassas I, lunde PKM. National drug policies: the need for drug utilization studies. Trends pharmacol sci. 1986;7:331-4. 
2. Nehru M, Kohli K, Kapoor B, Sadhotra P, Chopra V, Sharma R. Drug utilization study in outpatient ophyhalmology department of government medical college Jammu. JK Sciences. 2005;7:149-51.

3. WHO publication. What is drug utilization research and why is it needed? Introduction to drug utilization research. 2003;8.

4. Sharma R, Kapoor B, Verma U. Drug utilization pattern during pregnancy in North India. Indian $\mathrm{J}$ of Medical Sciences. 2006;60(7):277-87.

5. Jadhav PR, Moghe VV, Deshmukh YA. Drug utilization study in ophthalmology outpatients at a tertiary care teaching hospital, ISRN Pharmacology, Article ID 768792. 2013;22.

6. W.H.O Geneva. How to investigate drug use in health facilities EMD Research. 1993;7:3-27.

7. Ajapuje P, Dhengre P, Giri VC, Khakse GM. Drug Prescription Practices among Pediatric Patients in Yavatmal, Central India. International Journal of Recent Trends in Science and Technology, ISSN 2277-2812 E-ISSN 2249-8109. 2012;5(2):104-6.

8. Sanz EJ, Boada JN. Drug utilization by children in Tenerif Island, Spain. Eur J Clin Pharmacol. 1998;34:495-9.

9. Torvi JR, Dambal S. Drug prescription pattern in pediatric outpatient clinic in a tertiary hospital. Curr Pediatr Res. 2011;15(2):77-80.

10. Simpson GB, Choudary GN. Comparative Analysis of Prescription Writing by Teaching and Non - Teaching Clinicians in and around Guntur. International Journal of Recent Trends in Science and Technology, ISSN 2277-2812 E-ISSN 2249-8109.2012;5(2):100-3.

11. Johnson AG, Seidmann P, Day RO. NSAID related adverse drug reaction interaction with clinical relevance: an update. Int $\mathrm{J}$ Clin pharmacol Ther. 1994;32(10);509-32.
12. Mohanty BK, Ashwini M, Hasamnis AA, Patil SS, Murty KSN, Jena SK. Prescription pattern the department of atertiary care hospital in Rajamundry, India. Journal of Clinical and Diagnostic Research. $2010 \mathrm{Feb}$;4):2047-51.

13. Bajait CS, Pimpalkhute SA, Sontakke SD, Jaiswal KM, Dawri AV. Prescribing pattern of medicine in chronic kidney disease with special emphasis on phosphate binders. IJP. 2014:46:35-9.

14. Global Antibiotic Resistance Partnership (GARP) India Working Group. Rationalizing antibiotic use to limit antibiotic resistance in India Indian J Med Res. 2011;134:281-94.

15. World Health Day- 7 April 2011. Available at: http://www.who.int/world-healthday/ 2011/en/.

16. Bhatnagar T, Mishra CP, Mishra RN. Drug prescription practices: A household study in rural Varanasi’ Indian J Prev Soc Med. 2003;34(1,2).

17. Toppo M, Verma N, Sinha T, Agrawal S. Prescription analysis report in Chhattisgarh SHRC Indian J Pharmacol. 2004;31:151-3.

18. Tripathi KD. Appendix -1. List of Essentialmedicine. $6^{\text {th }}$ Edn. Jaypee Brothers. Reprint; 2010:903-907.

19. Mirza NY, Desai S, Ganguly B. Prescribing pattern in a pediatric out-patient department in Gujarat. Bangladesh J Phar. 2009;4:39-42.

Cite this article as: Chandra S, Khan IN,

Mateenudin M, Chandrakapure A, Maaz S, Mubin F. Drug utilization study in OPD of a tertiary care hospital in a rural area of Jalna, Maharashtra, India by using WHO prescribing indicators. Int J Basic Clin Pharmacol 2018;7:55-8. 